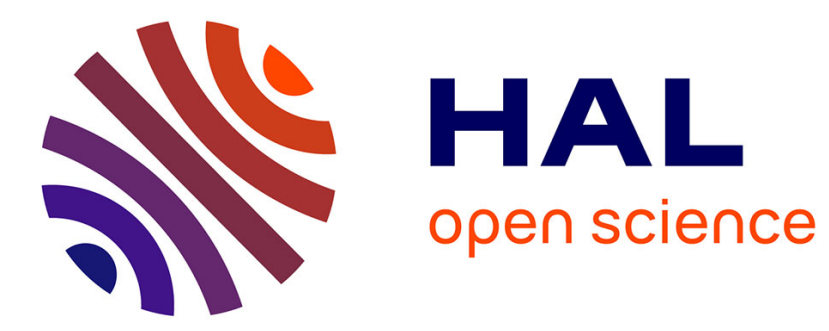

\title{
Les sciences sociales au prisme de l'alimentation (avant-propos)
}

\author{
Antoine Bernard de Raymond, Sylvain Parasie
}

\section{To cite this version:}

Antoine Bernard de Raymond, Sylvain Parasie. Les sciences sociales au prisme de l'alimentation (avant-propos). Terrains et Travaux: Revue de Sciences Sociales, 2005, n 9 (2), pp.3-11. hal02681426

\section{HAL Id: hal-02681426 \\ https://hal.inrae.fr/hal-02681426}

Submitted on 31 May 2020

HAL is a multi-disciplinary open access archive for the deposit and dissemination of scientific research documents, whether they are published or not. The documents may come from teaching and research institutions in France or abroad, or from public or private research centers.
L'archive ouverte pluridisciplinaire HAL, est destinée au dépôt et à la diffusion de documents scientifiques de niveau recherche, publiés ou non, émanant des établissements d'enseignement et de recherche français ou étrangers, des laboratoires publics ou privés. 


\title{
LES SCIENCES SOCIALES AU PRISME DE L'ALIMENTATION (AVANT-PROPOS)
}

\author{
Antoine Bernard de Raymond et Sylvain Parasie
}

ENS Cachan | Terrains \& travaux

\author{
$2005 / 2-n^{\circ} 9$ \\ pages 3 à 11
}

ISSN 1627-9506

Article disponible en ligne à l'adresse:

http://www.cairn.info/revue-terrains-et-travaux-2005-2-page-3.htm

Pour citer cet article :

de Raymond Antoine Bernard et Parasie Sylvain, «Les sciences sociales au prisme de l'alimentation (avant-propos) 》, Terrains \& travaux, 2005/2 n 9, p. 3-11.

Distribution électronique Cairn.info pour ENS Cachan.

(c) ENS Cachan. Tous droits réservés pour tous pays.

La reproduction ou représentation de cet article, notamment par photocopie, n'est autorisée que dans les limites des conditions générales d'utilisation du site ou, le cas échéant, des conditions générales de la licence souscrite par votre établissement. Toute autre reproduction ou représentation, en tout ou partie, sous quelque forme et de quelque manière que ce soit, est interdite sauf accord préalable et écrit de l'éditeur, en dehors des cas prévus par la législation en vigueur en France. II est précisé que son stockage dans une base de données est également interdit. 


\section{Les sciences sociales au prisme de l'alimentation}

(avant-propos)

Enquêtant sur les besoins des ménages ouvriers, Maurice Halbwachs s'interrogeait sur l'unité du besoin alimentaire dont témoignaient les enquêtes sociales, en se demandant s'il fallait dissocier « le besoin de nourriture de celui qui se rapporte à la façon dont les mets sont préparés et servis, à la quantité et à la qualité de la vaisselle, à l'emploi d'une nappe, et, pour les classes sociales plus élevées, aux divers ornements de la table, à l'éclairage de la salle à manger, au nombre des domestiques, etc. " (Halbwachs, 1970). Les frontières de l'alimentation semblent en effet difficiles à tracer scrupuleusement, et c'est à une conception large de l'alimentation que s'attache ce numéro de terrains \& travaux, puisque les différents articles réunis ici s'intéressent aussi bien aux façons de dresser la table, aux manières de table, qu'aux modes de production, aux sociabilités ou aux aliments proprement dits.

Qu'elles portent sur la consommation d'aliments, les goûts culinaires, les manières de table, la production des aliments ou les formes de sociabilité, les différentes contributions interrogent la situation alimentaire en mobilisant non seulement des méthodes et des objets divers, mais aussi des traditions et des disciplines différentes. Si bien que ce numéro peut se lire comme une invitation à parcourir l'histoire des sciences sociales à travers le prisme de l'alimentation. Si celle-ci occupe une place de choix dans les études sociologiques classiques, nous verrons qu'elle est aujourd'hui encore au cœur des renouvellements théoriques, et qu'elle pose question aux découpages disciplinaires traditionnels.

Sans vouloir forcer le trait excessivement, on peut différencier trois grandes perspectives théoriques parmi les articles réunis ici : une perspective classique qui voit dans l'alimentation une voie d'accès à des niveaux de vie et à des cultures de classe; une approche qui s'interroge sur la construction sociale des aliments; et une 
perspective plus anthropologique, qui étudie la situation alimentaire pour penser à nouveaux frais les liens entre natures et cultures.

\section{Une sociologie classique de l'alimentation}

Si l'on parcourt les principaux travaux de sociologie de la culture et des niveaux de vie, on constate que l'alimentation occupe une place centrale dans ces analyses. Depuis les enquêtes leplaysiennes jusqu'à Pierre Bourdieu, en passant par les travaux de Maurice Halbwachs et de Paul-Henri Chombart de Lauwe, l'alimentation au même titre que l'habillement et le vêtement - constitue un élément essentiel des dispositifs d'enquête sur la culture et les niveaux de vie. L'alimentation joue ici le rôle d'un révélateur de niveaux de vie et de cultures de classe.

Le tableau (Le gourmet) de G. Schalken qui illustre la couverture de La Distinction (Bourdieu, 1979) synthétise à lui seul ce que signifie l'alimentation pour la sociologie des niveaux de vie ou, dit autrement, ce qu'est une sociologie classique de l'alimentation. L'observation de ce que l'individu mange, et de la façon dont il le mange représente un outil privilégié pour accéder à une culture de classe. En quelque sorte, pour une sociologie classique de l'alimentation, cette dernière est l'expression d'une culture ou sert de support pour développer une théorie de la société ou du changement social.

Le sociologue se fait à ce titre l'héritier des enquêtes menées par les réformateurs sociaux qui, par leurs observations, entreprenaient de définir les besoins des classes laborieuses (le logement, l'habillement et l'alimentation dans la "loi d'Engel") et de trouver les meilleurs moyens d'y subvenir. Pour la sociologie des niveaux de vie, l'alimentation jouit d'un statut particulier non seulement parce qu'elle entre dans le cadre de cette "anthropologie des besoins" héritée de la mouvance réformatrice, mais aussi parce qu'elle touche très directement aux questions du logement et de la sociabilité. C'est pourquoi la sociologie urbaine des années 1950 et 1960 investit volontiers le terrain de l'alimentation, en examinant le cadre dans lequel sont pris les repas, et en tentant de prescrire une 
conformation du logement qui soit cohérente avec les besoins des ouvriers en matière de repas et de sociabilité.

Outre cette dimension d'ethnographie de l'alimentation replacée dans son milieu "écologique", cette sociologie classique de l'alimentation prête une attention particulière au "volume et à la structure" de la consommation d'aliments, pris comme des objets aux caractéristiques sociales bien définies. De ce fait, elle appelle à une analyse quantitative de la consommation alimentaire, pour faire du panier de biens le symbole d'un certain niveau de vie ou d'une certaine culture de classe. Dans une optique différente, cette sociologie quantitative de l'alimentation s'intéresse à la temporalité des repas, ou, plus largement, des prises alimentaires, et ce, moins souvent pour différencier des cultures de classe que pour établir des comparaisons internationales, et définir des "modèles alimentaires " propres à chaque pays étudié ${ }^{1}$. Plus largement, les comportements alimentaires apparaissent au cœur de quelques théories sociologiques majeures. Chez Chombart de Lauwe tout comme chez Bourdieu, l'alimentation constitue le socle d'une théorie des cultures de classe, différenciées par leur distance à la nécessité ${ }^{2}$. On pensera également à Norbert Elias, dont on sait combien l'enquête sur les manières de table à travers les manuels de savoir-vivre a nourri une théorie des déplacements des seuils de sensibilité, et donc une théorie ambitieuse du changement social.

À bien des égards, on pourrait lire certaines contributions de ce dossier comme le déplacement de cette sociologie classique vers de nouveaux territoires, de nouveaux objets, jusqu'alors inexplorés. Émilie Giret étudie ainsi comment, dans un milieu isolé et confiné les îles Kerguelen - l'alimentation est l'occasion pour des individus coupés de leur milieu habituel de redéfinir leur identité. L'angoisse

\footnotetext{
1 Cela est en parti dû au contexte dans lequel les enquêtes sur les emplois du temps ont été conçues, et sur lequel revient Thibaut de Saint Pol dans son article. On peut à bien des égards lire la contribution de Thibaut de Saint Pol dans ce volume comme un renouvellement de cette sociologie quantitative des temporalités de l'alimentation.

2 «Le véritable principe des différences qui s'observent dans le domaine de la consommation et bien audelà, est l'opposition entre les goûts de luxe (ou de liberté), et les goûts de nécessité : les premiers sont le propre des individus qui sont le produit de conditions matérielles d'existence définies par la distance à la nécessité [...] qu'assure la possession d'un capital ; les seconds expriment, dans leur ajustement même, les nécessités dont ils sont le produit. C'est ainsi que l'on peut déduire les goûts populaires pour les nourritures à la fois les plus nourrissantes et les plus économiques [...] de la nécessité de reproduire au moindre coût la force de travail qui s'impose, comme sa définition même, au prolétariat. " (Bourdieu, 1979, p. 198). Cf. aussi, Chombart de Lauwe, (1977).
} 
des personnes se concentre sur l'alimentation qui constitue à la fois un motif de revendication ethnique et un support de négociation d'une identité individuelle fragilisée.

Catherine Ferland présente ensuite un terrain jusqu'ici resté dans l'ombre - la culture de la bière en Nouvelle-France aux $17^{\mathrm{e}}$ et $18^{\mathrm{e}}$ siècles - en cherchant à comprendre comment s'est mise en place une culture matérielle associée à la production et à la consommation de bière.

Enfin, Thibaut de Saint Pol cherche à décentrer l'intérêt classique pour le contenu des repas vers la question de leurs temporalités, en s'appuyant sur une enquête quantitative (les enquêtes "emploi du temps » de l'INSEE).

Ces contributions apportent, chacune à leur manière, un profond renouvellement de la sociologie de l'alimentation. Une autre façon de renouveler la sociologie de l'alimentation consiste non pas ou non seulement à rechercher de nouveaux objets, mais à questionner les objets de la sociologie de l'alimentation. En effet, dire que telle ou telle denrée alimentaire est, par exemple, un "bien de luxe", suppose que l'on s'accorde sur la définition du bien en question, et que l'on se coordonne sur des normes et des critères de jugement.

\section{Enquête sur la construction sociale des aliments}

Or, un certain nombre d'analyses, notamment historiques, montrent que cela n'a rien d'évident, et qu'aboutir à une définition partagée d'un bien est souvent le résultat d'un processus long, qui réclame un investissement important de la part de nombreux acteurs. En effet, dans la première perspective que nous avons présentée, l'aliment, en tant qu'objet, ne pose pas en soi de problème : il est en quelque sorte déjà là, et l'on ne s'interroge pas sur son histoire, sur la façon dont il est devenu un aliment. Mais tout au long de ce parcours parfois chaotique, le produit, comme le fleuve qui emporte avec lui des alluvions, se charge d'éléments - régulations institutionnelles, classifications, dispositifs marchands mis en place par les professionnels, apprentissages réalisés par les consommateurs, etc. qui expliquent en grande partie ce que l'on est susceptible d'observer " en coupe synchronique ". 
Du coup, ces travaux en histoire (Kaplan, 2002; Stanziani, 2003 ; Stanziani, 2004) ou en économie des conventions (Allaire et Boyer, 1995 ; Casabianca et Valceschini, 1996) s'intéressent moins à la consommation de denrées alimentaires pour remonter plutôt en amont, vers la production, attendu que l'histoire de la constitution d'une offre de produits alimentaires permet de dessiner les contours de l'espace dans lequel se déploie une demande alimentaire. Ainsi les travaux issus de cette perspective en termes de " construction sociale du produit" abordent très souvent les situations de controverses autour des qualités d'un produit, les solutions légales, juridiques, et classificatoires qui sont trouvées à ces litiges, ou encore les dispositifs sociotechniques mis en place par les professionnels du marché pour construire et capter une demande marchande. Autrement dit, ces travaux sont plutôt des travaux de sociologie économique, et étudient souvent la façon dont la définition d'un produit ou de ses qualités reconfigure le jeu des acteurs sur le marché.

Dans ce dossier sur l'alimentation, on compte plusieurs contributions qui vont dans ce sens pour aborder les enjeux de définition, de classification et de circulation des produits. Ainsi, Céline Granjou et Egizio Valceschini montrent comment la mise en œuvre des textes légaux organisant la traçabilité dans les filières agro-alimentaires aboutissent à une revalorisation des "circuits courts" de distribution des produits, ce qui met le consommateur en contact direct avec le producteur.

Alors que de nombreux travaux de sociologie économique cherchent à comprendre comment la critique structure la demande, Pierre-Marie Chauvin montre ensuite comment les critères de classification définis par le critique vinicole américain Robert Parker réorientent le monde de production du vin de Bordeaux. Selon l'auteur, si le discours du critique trouve une prise sur la région de Bordeaux c'est parce qu'en réalité, pour novateurs qu'ils soient, les critères promus par Parker restent fortement orientés par les classifications traditionnelles des vins de Bordeaux.

En amont du processus de production lui-même, Philippe Rousselot étudie les mesures de la qualité des pâtes alimentaires construites par les ingénieurs, et observe que ce travail d'optimisation des 
qualités naturelles du produit (la pâte ou le blé qui sert à sa fabrication) est orienté, au moins implicitement, par une théorie du consommateur, qui influe fortement sur les qualités finales du produit.

Guilhem Anzalone se situe lui en aval de la production, pour appréhender les conditions dans lesquelles circule un produit (la viande), pour montrer que cette étape constitue un élément décisif de la "désanimalisation " et de la réification de la viande, qui sont des conditions nécessaires de sa marchandisation.

Enfin, Sylvain Leteux prolonge cette interrogation sur la construction sociale des aliments en se penchant sur le tabou de l'hippophagie dans la France du $19^{\text {e }}$ siècle. Cherchant à expliquer pourquoi la consommation de la viande de cheval, si elle est de plus en plus acceptée au 19e siècle - sous l'impulsion des hygiénistes, demeure très faible en France, l'auteur met l'accent sur la dimension économique de ce tabou, et sur les réactions corporatistes de la boucherie française.

\section{Natures, cultures et alimentation}

Au-delà des approches classiques en sociologie, pour lesquelles l'alimentation offre un accès à des niveaux de vie, à des cultures de classe et donc indirectement au changement social, et de la perspective de sociologie économique pour laquelle l'enjeu théorique réside avant tout dans la construction problématique d'un bien marchand à partir d'un bien naturel, on observe un déplacement récent des études sur l'alimentation. À bien des égards ce déplacement se nourrit d'une perspective plus nettement anthropologique. Elle consiste à voir dans le phénomène alimentaire une situation dans laquelle se négocient et se définissent simultanément des identités, individuelles et collectives, et des "natures". Contrairement à l'approche "constructiviste", il ne s'agit pas de comprendre comment on construit un bien marchand à partir d'un bien provenant de la "nature", mais de comprendre comment la définition de la "nature" se joue elle-même dans le phénomène alimentaire. 
"Où s'arrête la nature et où la culture commence-t-elle lorsque je prends un repas (...)?", se demande Philippe Descola lorsqu'il présente les fondements d'une "anthropologie de la nature" (Descola, 2002, p. 15). Il serait en effet trompeur de penser que la nature existe comme un tout cohérent que chaque culture interpréterait d'une façon plus ou moins juste. Chaque société se caractérise plutôt par un partage spécifique entre "humains" et "non humains" si bien qu'il serait plus pertinent, comme l'écrit Bruno Latour, de parler de "natures-cultures» (Latour, 1997, p. 143), c'est-à-dire d'autant de manières de construire simultanément une nature et une culture, d'ordonner la totalité des êtres.

L'alimentation, c'est ce que nous montrent les articles réunis dans la dernière partie de ce numéro, est au cœur de la construction de ces " natures-cultures ». Elle engage en effet simultanément, dans cette approche, des collectifs humains et une " nature " dont la cohérence et la solidité ne sont jamais totalement acquises, mais toujours faites d'arrangements et de négociations. Ce qui se dessine comme une approche nouvelle en sociologie de l'alimentation puise abondamment, on le voit, dans l'anthropologie et dans l'ethnologie : voilà un autre trait que partagent les articles qui peuvent se rattacher à cette approche.

Sait-on par exemple ce qu'est un " aliment naturel » ? Gaëlle Geffroy et Christian Papinot nous montrent ainsi comment des pêcheurs à pied bretons, qui ramassent des coquillages et des crustacés, développent une connaissance particulière et très fine d'un espace de collecte à la fois sauvage et domestique. Et dans le même temps où ils s'approprient cet espace "naturel ", l'activité de collecte est au cœur de la constitution et du renforcement de l'appartenance à la collectivité locale. Les auteurs s'appuient ensuite sur cette analyse d'une construction simultanée d'une " nature" et d'une "culture" pour comprendre les réticences des pêcheurs à l'égard des législations réglementant la collecte et la consommation: leur rapport particulier à cet espace les conduit à contester les décisions officielles sur les dangers sanitaires associés à la consommation des coquillages.

Est-on bien sûr de savoir faire la différence entre des aliments comestibles et des aliments non comestibles? Dans l'article qu'elle consacre à la pratique géophagique - le fait d’ingérer de la terre, ou 
plus exactement, ici, de l'argile - par des immigrées d'Afrique de l'Ouest à Paris, Giovanna Pessoa nous montre non seulement que la comestibilité n'est pas inscrite dans les propriétés physiques des aliments, mais que la consommation du kaolin engage simultanément une anthropologie de la nature et également la question du rapport entre les hommes et les femmes. En suscitant le trouble dans la classification des aliments, c'est très directement la question du pouvoir et sa dimension de genre qui est ainsi posée.

Catherine Rémy nous entraîne enfin dans un abattoir afin de comprendre comment l'animal destiné à être transformé en " objetviande " constitue l'enjeu des interactions entre les différents employés. Présentant le processus historique de séparation de l'activité de boucherie et d'abattage, l'auteure se demande comment interagissent les travailleurs de l'abattoir alors que l'origine animale de la viande ne peut être facilement oubliée. Cette ethnographie, qui se veut très attentive à l'action en train de se faire, montre ainsi comment les "tueurs" se distinguent des "non-tueurs" dans les interactions en apparence les plus ordinaires. Là encore, comme dans les autres contributions réunies dans la troisième partie de ce numéro, on mesure à quel point des identités se négocient et se construisent simultanément à la construction d'une " nature ".

\section{RÉFÉRENCES}

ALLAIRE (G.) et BOYER (R.), 1995. La Grande transformation de l'agriculture: lectures conventionnalistes et régulationnistes, Paris, INRA-Economica.

BOURDIEU (P.), 1979. La Distinction, Paris, Minuit.

CASABIANCA (F.) et VALCESCHINI (E.), 1996. Construction sociale de la qualité, Paris, INRA.

CHOMBART de LAUWE (P.-H.), 1977. La Vie quotidienne des familles ouvrières, Paris, Éd. du CNRS.

DESCOLA (P.), 2002. "L'anthropologie de la nature ", Annales HSS, janvier-février, ${ }^{\circ} 1$, pp. 9-25.

ELIAS (N.), (1991), La Dynamique de l'occident, Paris, CalmannLévy. 
HALBWACHS (M.), 1970. La Classe ouvrière et les niveaux de vie. Recherches sur la hiérarchie des besoins dans les sociétés industrielles contemporaines, Paris, Gordon et Breach (1ère édition : 1913).

LATOUR (B.), 1997. Nous n'avons jamais été modernes. Essai d'anthropologie symétrique, Paris, La Découverte.

STANZIANI (A.) (dir.), 2003, La Qualité des produits en France (XVIII ${ }^{e}$ XX ${ }^{e}$ siècles), Paris, Belin.

STANZIANI (A.), 2004. Histoire de la qualité alimentaire, Paris, Seuil. 